Return to the Manage Active Submissions page at http://spie.org/submissions/tasks.aspx and approve or disapprove this submission. Your manuscript will not be published without this approval. Please contact author_help@spie.org with any questions or concerns.

\title{
Cross-calibration of the Oceansat-2 Ocean Colour Monitor (OCM) with Terra and Aqua MODIS
}

\author{
Amit Angal $^{\mathrm{a}}$, Jake Brinkmann ${ }^{\mathrm{a}}$, A. Senthil Kumar ${ }^{\mathrm{b}}$, Xiaoxiong Xiong ${ }^{\mathrm{c}}$ \\ ${ }^{a}$ Science Systems and Applications Inc. 10210 Greenbelt Rd, Lanham, MD 20706 \\ ${ }^{b}$ Indian Institute of Remote Sensing, ISRO, Department of Space, Kalidas Rd, Dehradun, India \\ 248001
}

${ }^{c}$ Sciences and Exploration Directorate, NASA/GSFC, Greenbelt, MD 20771

\begin{abstract}
The Ocean Colour Monitor (OCM) sensor on-board the Oceansat-2 spacecraft has been operational since its launch in September, 2009. The Oceansat 2 OCM primary design goal is to provide continuity to Oceansat-1 OCM to obtain information regarding various ocean-colour variables. OCM acquires Earth scene measurements in eight multi-spectral bands in the range from 402 to $885 \mathrm{~nm}$. The MODIS sensor on the Terra and Aqua spacecraft has been successfully operating for over a decade collecting measurements of the earth's land, ocean surface and atmosphere. The MODIS spectral bands, designed for land and ocean applications, cover the spectral range from 412 to $869 \mathrm{~nm}$. This study focuses on comparing the radiometric calibration stability of OCM using nearsimultaneous TOA measurements with Terra and Aqua MODIS acquired over the Libya 4 target. Same-day scenepairs from all three sensors (OCM, Terra and Aqua MODIS) between August, 2014 and September, 2015 were chosen for this analysis. On a given day, the OCM overpass is approximately an hour after the Terra overpass and an hour before the Aqua overpass. Due to the orbital differences between Terra and Aqua, MODIS images the Libya 4 site at different scan-angles on a given day. Some of the high-gain ocean bands for MODIS tend to saturate while viewing the bright Libya 4 target, but bands $8-10(412 \mathrm{~nm}-486 \mathrm{~nm})$ provide an unsaturated response and are used for comparison with the spectrally similar OCM bands. All the standard corrections such as bidirectional reflectance factor (BRDF), relative spectral response mismatch, and impact for atmospheric water-vapor are applied to obtain the reflectance differences between OCM and the two MODIS instruments. Furthermore, OCM is used as a transfer radiometer to obtain the calibration differences between Terra and Aqua MODIS reflective solar bands.
\end{abstract}

\section{Introduction}

The MODIS sensors onboard the Terra and Aqua spacecraft have been operating successfully since their launch in December 1999 and May 2002. The reflective solar bands (RSB) of the MODIS instruments are used in numerous science products and an accurate on-orbit calibration is essential to maintain the high quality of these science products. The on-orbit calibration for the RSB is performed using regular solar diffuser (SD) and lunar measurements with supplemental input from desert sites to characterize the scan-angle dependence [1]. Band 8 (412 $\mathrm{nm})$ and Band $9(443 \mathrm{~nm})$ are the high-gain ocean bands primarily used in the various ocean-colour applications. A long-term stability of the reflectances derived by these bands is critical in supporting various ocean colour products. Also, calibration comparisons with spectrally matching bands on other satellite sensors are beneficial to assess the consistency of multi-sensor ocean colour products.

The Oceansat-2 Ocean Colour Monitor (OCM) sensor has been operational since September 2009 and is used in the retrieval of various ocean-colour variables in the spectral range from 402 to $885 \mathrm{~nm}$. The spectral bands of OCM have a similar spectral coverage as the MODIS ocean-colour bands as both sensors support ocean-colour applications. After undergoing an extensive prelaunch characterization, the in-flight system degradation of OCM is monitored using four LEDs in each band [2], [3], [4], [5]. Near-simultaneous overpasses with MODIS over CEOSendorsed ground targets present a unique opportunity to evaluate the inflight consistency of the OCM spectral bands. In this study, level 1B (radiometrically calibrated) granules from OCM and MODIS sensors over Libya 4 are selected for comparison. The top-of-atmosphere reflectances, with appropriate corrections, are compared to evaluate the differences between the MODIS and OCM sensors. 
Return to the Manage Active Submissions page at http://spie.org/submissions/tasks.aspx and approve or disapprove this submission. Your manuscript will not be published without this approval. Please contact author_help@spie.org with any questions or concerns.

MODIS covers a wide-swath of $2330 \mathrm{~km}$ via a two-sided scan-mirror. The reflectance of the scan mirror is dependent on the angle of incidence (AOI) and is characterized by a response versus scan-angle (RVS) function. Significant challenges are experienced in the on-orbit calibration of the short-wavelength visible (VIS) bands, especially in Terra MODIS [6]. Due to the different orbits of Terra and Aqua satellites, the Libya 4 site is observed at different AOIs on a given day. Comparisons with OCM present a unique opportunity to compare the Terra and Aqua reflectances, measured at different AOI, and therefore provide an assessment of the effectiveness of the scanangle correction on-orbit. Section 2 presents a brief overview of the MODIS and OCM sensors and the Libya 4 site. The methodology is described in Section 3 and Section 4 discusses the Results and Analysis. Section 5 discusses the conclusions and also identifies future work.

\section{a. MODIS and OCM sensor}

\section{Sensor and Site Overview}

The eight spectral bands of OCM cover the spectral range from 402 to $885 \mathrm{~nm}$. Unlike the MODIS instruments that orbit at $705 \mathrm{~km}$, Oceansat-2 orbits at $720 \mathrm{~km}$ with a revisit cycle of 2 days. Due to its smaller swath $(1440 \mathrm{~km})$ compared to the $2330 \mathrm{~km}$ MODIS swath, it does not necessarily view every part of the earth every 2 days. OCM images the Libya 4 site once every 8 days. A summary of the spectral coverage of OCM bands is listed in Table I.

The RSB of both MODIS instruments are calibrated on-orbit using regular solar diffuser (SD) and near-monthly lunar measurements. While the SD measurements are primarily used to derive the detector gain $\left(\mathrm{m}_{1}\right)$, they are also used together with the lunar measurements to characterize the on-orbit response versus scan-angle (RVS) for the MODIS RSB. The $m_{1}$ and RVS together represent the gain of the instrument at any scan-angle. The MODIS viewing angle range $\left( \pm 55^{\circ}\right)$ is often represented in terms of angle of incidence (AOI) on the scan mirror, $\theta$, which ranges from 10.5 to $65.5^{\circ}$. The frame number $\mathrm{F}$ from the earth-view product and the AOI are related by,

$$
\theta=10.5^{\circ}+\left(65.5^{\circ}-10.5^{\circ}\right) * \mathrm{~F} / 1353
$$

where $\mathrm{F}=0,1,2 \ldots 1353$

The wide swath of MODIS allows a view of the Libya 4 site once every 2 to 3 days. However, these views are at different frame number (or AOI). Libya 4 or any other earth-view site is observed at a given frame number once every 16 days. MODIS has 20 RSB covering the spectral range from 410 to $2300 \mathrm{~nm}$ with each spectral band designed with a specific science application. In this study only the bands spectrally overlapping with OCM are considered and are summarized in Table I. While a majority of the bands listed are high-gain ocean bands, measurements from bands 1 and 2 can also considered.

Table I. Summary of the Spectral Coverage of the overlapping MODIS and OCM-2 bands

\begin{tabular}{c|c|c|c}
\hline \hline \multicolumn{2}{c|}{ Terra and Aqua MODIS (Spectral range in nm) } & \multicolumn{2}{c}{ Oceansat 2 OCM (Spectral range in nm) } \\
\hline \hline 8 & $405-420$ & 1 & $402-422$ \\
\hline 9 & $438-448$ & 2 & $433-453$ \\
\hline 10 & $483-493$ & 3 & $480-500$ \\
\hline 11 & $526-536$ & 4 & $500-520$ \\
\hline 12 & $546-556$ & 5 & $545-565$ \\
\hline 1 & $620-670$ & 6 & $610-630$ \\
\hline 15 & $743-753$ & 7 & $725-755$ \\
\hline 16,2 & $862-877,841-876$ & 8 & $845-885$ \\
\hline \hline
\end{tabular}

The bands 8 through 16 of both MODIS instruments are specifically designed for ocean colour applications and hence are commonly referred to as "ocean-bands". In order to accommodate the low signal levels of the ocean scenes, the gain for these bands is set at a higher level in comparison with the other MODIS RSB. As a result, these bands are prone to saturation while viewing high-radiance ground targets. Libya 4 being a high-radiance target, the bands $11,12,15$, and 16 exhibit partial or complete saturation. OCM, an ocean colour monitoring sensor, also has a higher gain setting for these bands to accommodate a low signal levels from ocean. Hence, the OCM bands beyond 


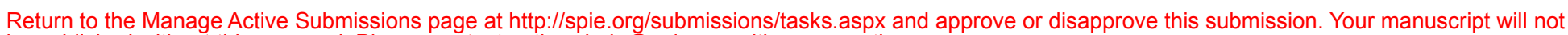
be published without this approval. Please contact author_help@spie.org with any questions or concerns.

$500 \mathrm{~nm}$ also saturate while viewing the Libya 4 target. Hence the MODIS bands 8 and 9 with the spectrally matching OCM bands are the only bands considered in this study.

\section{b. Libya 4 ground target:}

Libya $4\left(+28.55^{\circ},+23.39^{\circ}\right)$ is a CEOS-endorsed calibration/validation site widely used for sensor intercomparison. The temporal, spatial, and spectral stability of Libya 4 is well known, making it an ideal candidate to perform intercomparison of the OCM and MODIS sensors, which view the site at different times of the day [7],[8]. Figure 1 shows the OCM overpass over Libya 4 overlapped with a Terra MODIS overpass from August, 2015. OCM band 1 and MODIS band 8 have been used to produce this image.

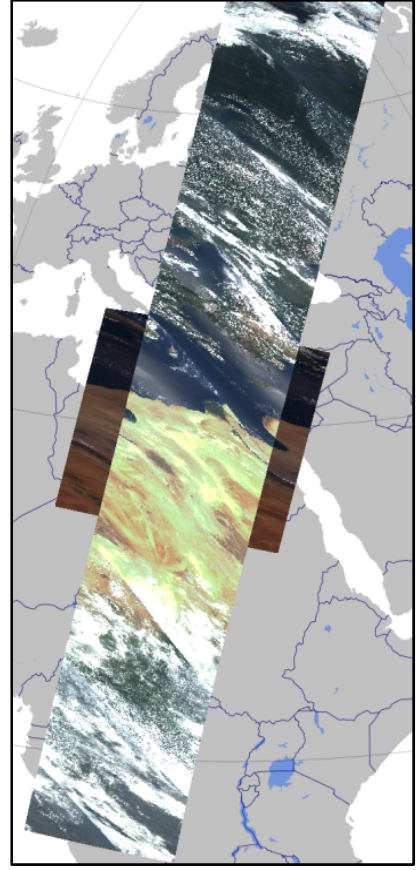

(a)

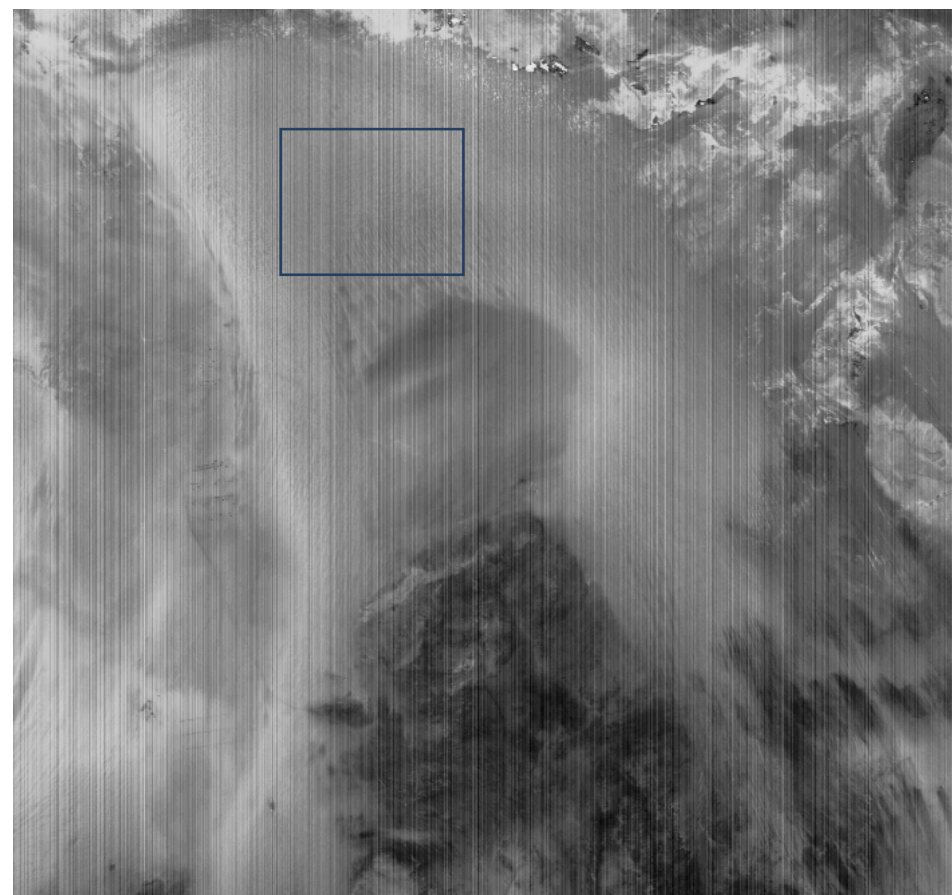

(b)

Figure 1 (a) OCM and Terra MODIS overpass over Libya 4 from August, 2015. (b) A close-up view of Libya 4 as observed by OCM band $1(412 \mathrm{~nm})$.

Since OCM is a pushbroom sensor, the presence of residual striping in the calibrated image is unsurprising. The blue box denotes the region of interest (ROI) $[20 \times 20 \mathrm{~km}]$ chosen for this study and has been recommended after intensive efforts from CNES [7]. The residual striping observed in the images bears minimal impact on the average TOA reflectance computed over the ROI.

\section{Methodology}

\section{a. Data Product Details:}

The OCM Level 1B (L1B) radiance products were obtained free of cost through the web [9]. The Global Area Coverage (GAC) data is sub sampled to $1 \mathrm{~km} \times 1 \mathrm{~km}$ spatial resolution and obtained in HDF4 format. The OCM camera is mounted with tilt of +20 and -20 degrees to avoid sun glint [9]. From May to August, the tilt is +20 degrees and from September to April, the tilt is -20 degrees. Although data has been collected since its launch in September 2009, only the OCM data after July, 2014 has been considered in this study. At present, archival of OCM GAC data is available for 10 months of data at a given time. Since the aim of the present study is to develop an operational methodology to cross calibrate the two sensors, only the data available for free download is considered. Once it is established, it is proposed to use long term trend variation of biases, if any. 
Return to the Manage Active Submissions page at http://spie.org/submissions/tasks.aspx and approve or disapprove this submission. Your manuscript will not be published without this approval. Please contact author_help@spie.org with any questions or concerns.
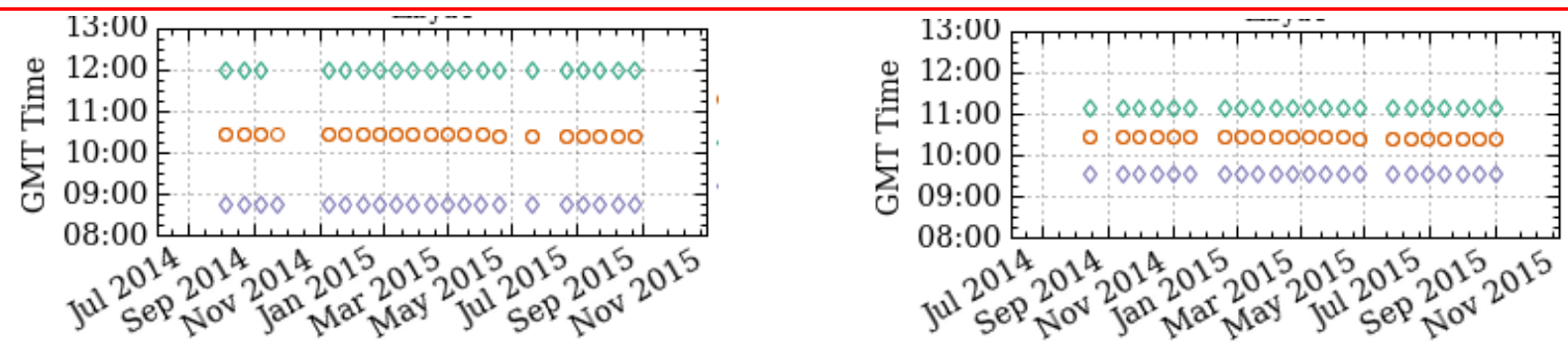

Figure 2a Libya 4 overpass times for OCM (red), Aqua MODIS (green) and Terra MODIS (purple). The Terra MODIS measurements are acquired around frame 1133 (end of scan) and Aqua MODIS measurements are acquired around frame 1245. Figure 2b. Libya 4 overpass times for OCM (red), Aqua MODIS (green) and Terra MODIS (purple). The Terra MODIS measurements are acquired around frame 178 (start of scan) and Aqua MODIS measurements are acquired around frame 326.

\section{b. TOA reflectance calculation:}

The commonly selected ROI, discussed earlier, is used to compute the band-specific TOA reflectance for every overpass. In the case of Terra and Aqua MODIS, the TOA reflectance is computed using the reflectance_scales and reflectance_offsets provided in the MODIS L1B granules. In the case of OCM, the L1B GAC granules report scaled radiance (scaling factor of 10), which is then converted to TOA reflectance using the band-specific exo-atmospheric solar irradiance values (ESUN). The L1B GAC granules use the calibration coefficients derived during preflight characterization. An on-orbit assessment of these preflight characterization coefficients was performed using five lunar observations. The lunar observations conducted between July 2010 and July 2013 were used to normalize with respect to the ROLO model results and on-orbit calibration coefficients were derived. The results revealed that the retrieved L1B radiances from OCM need to be scaled by a factor, to reflect the sensor changes on-orbit. These factors reduce the radiances for OCM band 1 by about $5 \%$ and by about $24 \%$ for OCM band 2 . The results presented in this study include the OCM reflectance corrected using this scaling factor [9].

\section{c. BRDF correction}

Due to the difference in the overpass times, the changes in the surface and atmospheric bi-directional reflectance function (BRDF) need to be considered. A semi-empirical BRDF model [10] is used to derive the BRDF normalized reflectances for Terra and Aqua MODIS. Using the first 3-years of MODIS measurements over the Libya 4 site, a semi-empirical BRDF consisting of two kernel-driven components was used. This kernel-driven BRDF model at a given wavelength is expressed as follows:

$$
\hat{\rho}=k_{0}+k_{1} f_{1}\left(\theta_{s}, \theta_{v}, \phi\right)+k_{2} f_{2}\left(\theta_{s}, \theta_{v}, \phi\right)
$$

where $\theta \mathrm{s}, \theta \mathrm{v}$, and $\boldsymbol{\Phi}$ are the solar zenith, view zenith, and relative azimuth angles, f1 is derived from the volume scattering component, and $\mathrm{f} 2$ is the component from surface scattering and geometric shadow casting theory. Coefficients $\mathrm{k}_{0}, \mathrm{k}_{1}$, and $\mathrm{k}_{2}$ are given as functions of parameters related to the physical structure and optical properties of our reflecting surface in the original model. Table I summarizes the BRDF fitting coefficients for Terra and Aqua MODIS band 8. The coefficients from Aqua MODIS are applied to OCM TOA reflectances to obtain BRDFcorrected reflectances. This BRDF correction also enables the comparison between Terra and Aqua MODIS that have a significant difference in the overpass times.

Table I. BRDF fitting coefficients for band 8 (412 nm)

\begin{tabular}{|c|c|c|c|c|}
\hline & \multicolumn{2}{|c|}{ Terra MODIS } & \multicolumn{2}{c|}{ Aqua MODIS } \\
\hline & Frame 179 & Frame 1133 & Frame 326 & Frame 1245 \\
\hline $\mathrm{k}_{0}$ & 0.2187 & 0.2240 & 0.2169 & 0.2298 \\
\hline $\mathrm{k}_{1}$ & -0.0259 & -0.0190 & -0.0336 & -0.0105 \\
\hline $\mathrm{k}_{2}$ & 0.7586 & 0.5036 & 0.6990 & 0.5295 \\
\hline
\end{tabular}


Return to the Manage Active Submissions page at http://spie.org/submissions/tasks.aspx and approve or disapprove this submission. Your manuscript will not be published without this approval. Please contact author_help@spie.org with any questions or concerns.

\section{d. Correction for RSR mismatch:}

Although the MODIS and OCM bands are closely matched in center-wavelengths, the relative spectral response (RSR) for the spectrally matching bands differs. A correction for this mismatch is essential before a reflectancecomparison between the two sensors is performed. Figure 3 shows the RSRs for the OCM bands 1 and 2 with the spectrally matching MODIS bands 8 and 9. Also plotted in red is the lifetime [August 2002-December 2010] average of TOA reflectance profile from ENVISAT-Sciamachy, a hyperspectral sensor. Using this hyperspectral information, the spectral band adjustment factors (SBAFs) are computed for the OCM bands 1 and 2. The Sciamachy reflectance spectrum shows very little variation across the OCM and MODIS RSR. Unlike the MODIS land/atmosphere bands, the ocean bands have a relatively narrow RSR therefore minimizing the SBAF impact. The SBAFs derived for both the bands are 0.998 for band 1 and 0.999 for band 2 therefore indicating a very little contribution. The correction for this RSR mismatch is considered in the subsequent calculations.
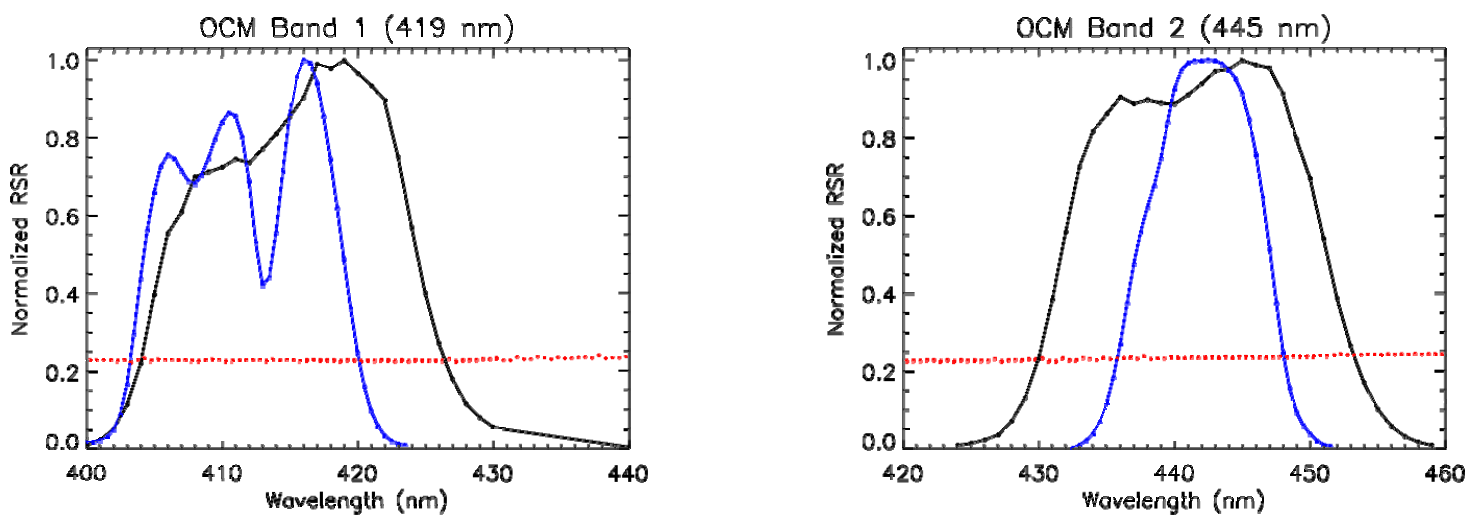

Figure 3a. Relative Spectral response for OCM band 1 (black) and Terra MODIS band 8 (blue). The red curve is the TOA reflectance retrieved by a hyperspectral sensor (ENVISAT-SCiamachy). b. Relative responses for OCM band 2 (black) and Terra MODIS band 9 (blue). The Aqua MODIS RSR are near-identical to the Terra MODIS RSRs and are not plotted here.

\section{a) Reflectance comparisons}

\section{Results and Analysis}

Using the procedure described in the previous section, the TOA reflectance for each spectral band is derived for the three sensors. Figure 4 shows the TOA reflectances using the selected same-day scenes. As discussed earlier, the MODIS measurements at the specific frame are temporally separated by 16 days (repeat cycle at the specific frame). In comparison with the results at small frames, the MODIS TOA reflectance exhibits more deviation (or spread) at large frame numbers. In order to account of the overpass time and view-geometry differences, a BRDF correction is essential for a meaningful comparison. Using the BRDF normalization coefficients from Table I, the BRDF normalized reflectance was obtained for the MODIS bands. The coefficients listed for Aqua MODIS were applied to generate the BRDF-normalized reflectance for OCM. The ratio of the BRDF-normalized reflectance $\rho_{\text {MODIS(brd-nrom) }} /$ $\rho_{\mathrm{OCM}(\mathrm{brd} f-\mathrm{norm})}$ has been shown in Figure $5 \mathrm{a}$ and $5 \mathrm{~b}$. The Aqua MODIS and OCM agreement (deviation from unity) for the $412 \mathrm{~nm}$ band varies between 5 and $20 \%$ and the Terra MODIS and OCM agreement varies between 5 and $30 \%$. The $443 \mathrm{~nm}$ band-pair exhibit better agreement with differences ranging from 2-10\% for Terra MODIS and 5$10 \%$ for Aqua MODIS (excluding the outliers). The deviation of these ratios from unity is a result of a combination of several factors: 1) RSR mismatch between the spectral bands of OCM and MODIS, 2) calibration uncertainties associated with each sensor, and 3) uncertainties associated with the cross-calibration process, i.e. the BRDF model and atmospheric impacts. As discussed in the previous section, the SBAFs for both the band-pairs are within 0.3\% and bear minimal impact on the observed differences. 
Return to the Manage Active Submissions page at http://spie.org/submissions/tasks.aspx and approve or disapprove this submission. Your manuscript will not be published without this approval. Please contact author_help@spie.org with any questions or concerns.
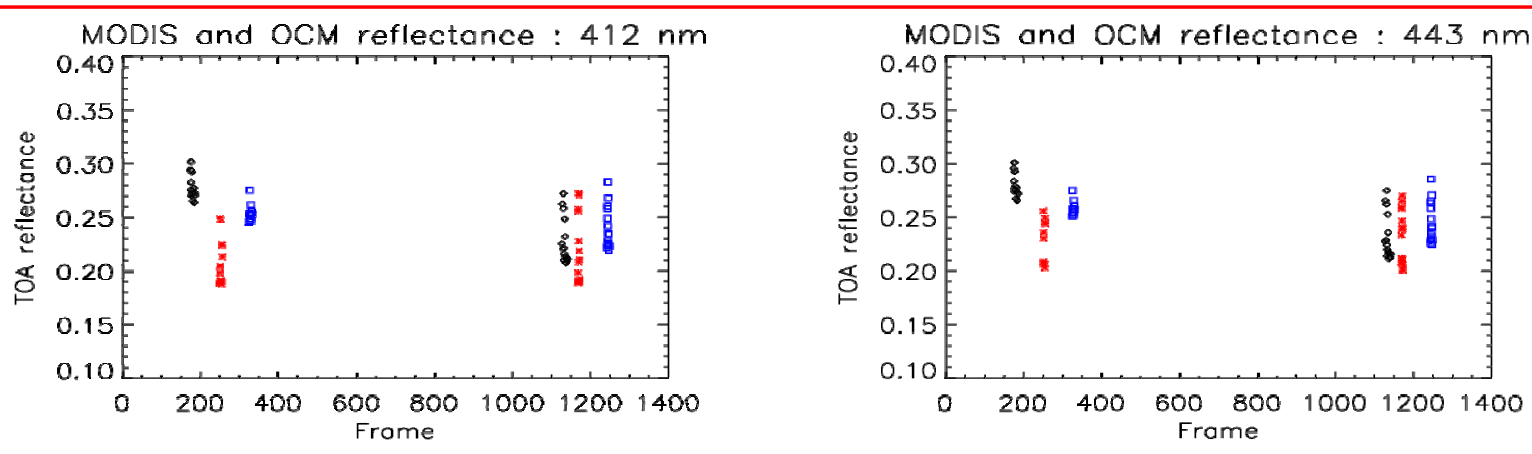

Figure 4a TOA reflectance at $412 \mathrm{~nm}$ for OCM [red] and MODIS [black: Terra, Blue: Aqua] sensors plotted as a function of MODIS frame (equivalent angle of incidence). 4b. TOA reflectance at $443 \mathrm{~nm}$ for OCM [red] and MODIS [black: Terra, Blue: Aqua] sensors plotted as a function of MODIS frame (equivalent angle of incidence).
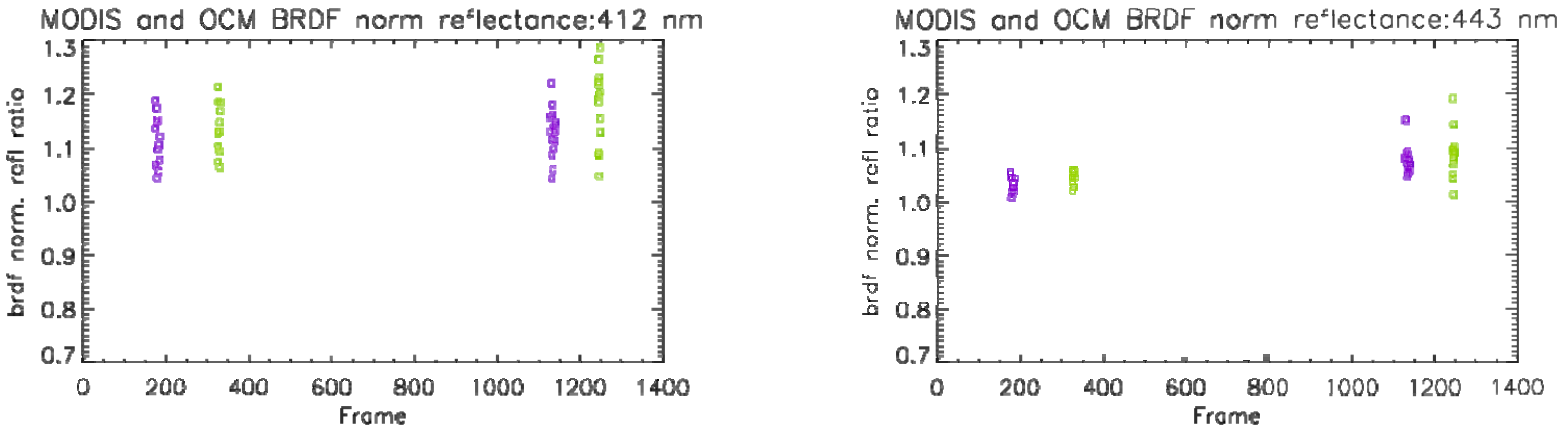

Figure 5a BRDF coefficients listed in Table I are applied to the MODIS and OCM data. The ratio of the BRDF normalized reflectances is plotted for $412 \mathrm{~nm}$ band as a function of MODIS frame (equivalent angle of incidence). [purple: Terra MODIS/OCM and green: Aqua MODIS/OCM) 5b. Similar plot for the $443 \mathrm{~nm}$ band-pair.
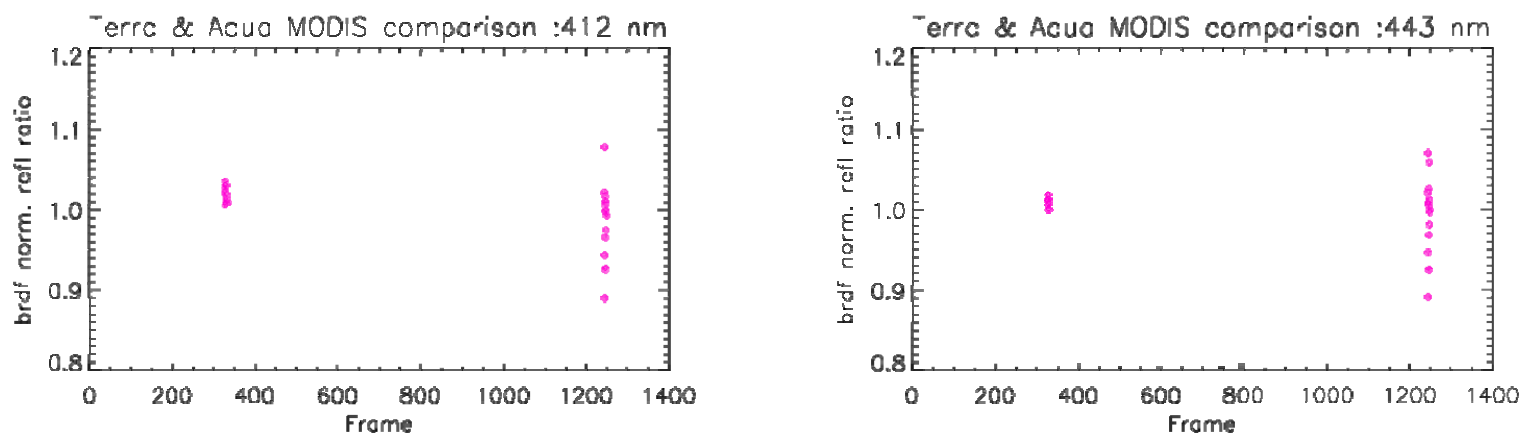

Figure 6a. BRDF normalized reflectance ratio between Terra and Aqua MODIS (412 nm). The ratio values shown at frame 326 have been computed using the Terra reflectance at frame 179 and Aqua reflectance at frame 326 . The ratio values shown at frame 1245 have been computed using the Terra reflectance at 1133 and Aqua reflectance values at frame 1245. Figure 6b. Similar results shown for the $443 \mathrm{~nm}$ channel of Terra and Aqua MODIS.

To further understand the reason behind these differences, a comparison of BRDF-normalized reflectances derived from Terra and Aqua MODIS is shown in Figure 6. Since Terra and Aqua MODIS have similar RSR, a SBAF correction is not required. A reasonable agreement $(<3 \%)$ is observed at the beginning of the scan [frame 326] for both the 412 and $443 \mathrm{~nm}$ bands. In comparison, the results observed at the end of scan, show differences of up to $10 \%$ for both the bands. MODIS acquires earth-scene images via a two-sided scan mirror at $1 \mathrm{~km}$ spatial resolution for 412 and $443 \mathrm{~nm}$ bands. Although the calibration is performed on a band and mirror-side basis, residual mirror side differences are observed, especially in Terra MODIS, due to well-understood reasons [11]. Also for a given 
Return to the Manage Active Submissions page at http://spie.org/submissions/tasks.aspx and approve or disapprove this submission. Your manuscript will not be published without this approval. Please contact author_help@spie.org with any questions or concerns.

acquisition, the $20 \times 20 \mathrm{~km}$ site may not be sampled uniformly by the two mirror sides. A larger ROI is expected to mitigate the impacts of these differences and will be considered for future enhancements. While significant differences are observed in the $412 \mathrm{~nm}$ channel between OCM and MODIS instruments, an intensive uncertainty analysis is warranted for a further understanding of these results.

\section{Conclusions and Future Work}

Since its launch in September, 2009 the OCM has been collecting scientific measurements used to derive various ocean colour variables. Same-day L1B scenes from OCM over Libya 4 were compared with Terra and Aqua MODIS. Due to saturation observing the Libyan desert, the comparison was restricted to 412 and $443 \mathrm{~nm}$ channels. In order to account for the surface and atmospheric BRDF effects, model coefficients were derived from the first 3 years of MODIS observations. Corrections for RSR mismatch were derived using hyperspectral measurements from ENVISAT Sciamachy. In comparison with the $412 \mathrm{~nm}$ band, a better agreement is observed in the $443 \mathrm{~nm}$ band. The Aqua MODIS and OCM agreement for the $412 \mathrm{~nm}$ band varies between 5 and $20 \%$ and the Terra MODIS and OCM agreement varies between 5 and $30 \%$. The $443 \mathrm{~nm}$ band-pair exhibit better agreement with differences ranging from $2-10 \%$ for Terra MODIS and $5-10 \%$ for Aqua MODIS. A more intensive analysis on various uncertainties associated with this comparison is planned in the future.

An operational methodology to cross-calibrate the MODIS sensors with OCM has been developed. Work is underway to acquire the entire time-series of data the OCM collects over Libya 4. In addition to evaluating OCM's long-term calibration stability, this data-set will also facilitate an evaluation of multi-year differences between the MODIS and OCM sensors. Since a majority of the MODIS and OCM bands saturate while viewing the Libya 4 site, it is imperative to choose a target (ocean) to extend the comparison to the other bands.

\section{Acknowledgements}

The authors would like to thank Xu Geng of MCST for assistance with the BRDF correction. The authors would also like to thank Ben Scarino, SSAI and David Doelling, NASA Langley for providing the Sciamachy data.

\section{References}

[1] Xiong, X., M. D. King, V. Salomonson, W. Barnes, B. N. Wenny, A. Angal, A. Wu, S. Madhavan, and D. Link, "Moderate Resolution Imaging Spectroradiometer on Terra and Aqua Missions", John Wiley \& Sons, Ltd, vol. 9781118945179, pp. 53-89, 2015.

[2] Babu, K.N, A. K. Shukla, V. N. Sridhar, Ajai, B. Damiri, F.J.O. Reyes, "Post-launch calibration of Ocean Colour Montor 2 using Kavaratti CAL-VAL site observations", Current Science, Vol 104, No. 10, Jan 2013

[3] Sridhar, V.N., K. B. Mehta, R.P. Prajapati, K. N. Babu, N. M. Suthar and A. K. Shukla, "Absolute vicarious calibration of OCM2 and AWiFS sensors using a reflectance-based method over land sites in the Rann of Kutch, Gujrat", International Journal of Remote Sensing, Vol. 34, Iss. 16, 2013

[4] Tholkapiyan M, Shanmugam P, Chauhan P, Suresh M, "Derivation of Calibration Coefficients for OCM-2 Sensor for Coastal Waters", J Geophys Remote Sensing, vol 1, issue 2, 2012

[5] A. Senthil Kumar, T. Radhika, P.K. Saritha, V. Keerthi, R. N. Anjani, M. Suresh Kumar, K. S. Sekhar, P. Satyanarayana, M. S. Naga Sudha, M. V. R. Sesha Sai, V. K. Dadhwal, "Generation of Vegetation Fraction and Surface Albedo Products Over India from Ocean Colour Monitor (OCM) Data Onboard Oceansat-2", Journal of the Indian Society of Remote Sensing, Volume 42, Issue 4, pp 701-709, 2014

[6] Sun, J., X. Xiong, A. Angal, H. Chen, A. Wu, and X. Geng, "Time-Dependent Response Versus Scan Angle for MODIS Reflective Solar Bands", IEEE Transactions on Geoscience and Remote Sensing, vol. 52, issue 6, pp. 31593174, 2014. 
Return to the Manage Active Submissions page at http://spie.org/submissions/tasks.aspx and approve or disapprove this submission. Your manuscript will not be published without this approval. Please contact author_help@spie.org with any questions or concerns.

[7] S. Lachérade, B. Fougnie, P. Henry, and P. Gamet, "Cross-calibration over desert sites: Description, methodology and operational implementation,"IEEE Trans. Geosci. Remote Sens., vol. 51, no. 3, pp. 1098-1113, Mar. 2013.

[8] Angal, A., X. Xiong, A. Wu, G. Chander, and T. Choi, "Multitemporal Cross-Calibration of the Terra MODIS and Landsat 7 ETM+ Reflective Solar Bands", IEEE Transactions on Geoscience and Remote Sensing, vol. 51, issue 4, pp. 1870-1882, 2013.

[9] http://218.248.0.134:8080/OCMWebSCAT/html/controller.jsp

[10] Roujean, J-L. and M. Leroy, "A Bidirectional Reflectance Model of the Earth's Surface for the Correction of Remote Sensing Data", J. of Geophysical Research, Vol. 97, No. D18, pp. 20,455-20,468, 1992

[11] Wu, A., X. Geng, A..E.Wald, and X. Xiong, "Tracking Terra MODIS on-orbit polarization sensitivity using pseudo-invariant desert sites ", Proc. SPIE 9639, Sensors, Systems, and Next-Generation Satellites XIX, 963914, 2015. 